\title{
Comunidades de abelhas Euglossina (Hymenoptera, Apidae) em fragmentos de Mata Atlântica no Sudeste do Brasil
}

\author{
André Villaça Ramalhoํㅜㄹ Maria Cristina Gaglianone ${ }^{1} \&$ Marcio Luiz de Oliveira $^{2}$
}

${ }^{1}$ Universidade Estadual do Norte Fluminense Darcy Ribeiro, Laboratório de Ciências Ambientais, Av. Alberto Lamego, 2000, 28015-602

Campos dos Goytacazes-RJ, Brasil. andre_villaa@yahoo.com.br; mcrisgag@uenf.br

${ }^{2}$ Instituto Nacional de Pesquisas da Amazônia, Coordenação de Pesquisas em Entomologia, Manaus, AM.

\begin{abstract}
Euglossine bee (Hymenoptera, Apidae) community in Atlantic Forest fragments in southeastern Brazil. The Euglossine bee community was sampled with chemical bait traps throughout 12 months (November 2004 to October 2005) in five remnants of submontane Atlantic Forest in São João river basin, in the north of Rio de Janeiro state with different sizes and degradation levels: Reserva Biológica União (3126 ha), Andorinhas (145 ha), Imbaú (130 ha), Estreito (21 ha) e Afetiva (19 ha). 4094 individuals belonging to 17 species of three genera (Euglossa, Eulaema and Exaerete) were captured. The species with highest values of relative abundance were Euglossa cordata (Linnaeus, 1758), Eulaema cingulata (Fabricius, 1804), Eulaema nigrita Lepeletier, 1841 and Euglossa sapphirina Moure, 1968, the last one being more important in the smaller remnants. Among the collected species Euglossa analis Westwood, 1840 is suggested as possible indicator of preserved forests. Comparing the five areas, positive and significant correlations were observed, bee species richness with area size and bee diversity $\left(\mathrm{H}^{\prime}\right)$ with floristic diversity $\left(\mathrm{H}^{\prime}\right)$. These results suggest that losses in forest size and habitat quality influence euglossine bee communities negatively by reducing the abundance and richness of species. The highest similarity values were observed in the Imbaú region remnants, distant from each other by up to $2 \mathrm{~km}$, suggesting that these areas are not isolated for euglossine populations, or they have been suffering similar fragmentation effects.
\end{abstract}

KEYWORDS. Atlantic Forest; orchid bees; bioindicator; forest fragmentation.

\begin{abstract}
RESUMO. Comunidades de abelhas Euglossina (Hymenoptera, Apidae) em Fragmentos de Mata Atlântica no sudeste do Brasil. A comunidade de abelhas Euglossina foi amostrada através de armadilhas com iscas aromáticas, ao longo de 12 meses (novembro de 2004 a outubro de 2005) em cinco fragmentos de Floresta Atlântica submontana com diferentes tamanhos e níveis de degradação, na bacia do Rio São João, norte do estado do Rio de Janeiro: Reserva Biológica União (3126 ha), Andorinhas (145 ha), Imbaú (130 ha), Estreito (21 ha) e Afetiva (19 ha). Foram registrados 4094 indivíduos pertencentes a 17 espécies de três gêneros (Euglossa, Eulaema e Exaerete) nas 5 áreas. As espécies com maior abundância relativa foram Euglossa cordata (Linnaeus, 1758), Eulaema cingulata (Fabricius, 1804), Eulaema nigrita Lepeletier, 1841 e Euglossa sapphirina Moure, 1968, sendo maior a importância relativa desta última nos fragmentos menores. Dentre as espécies encontradas, Euglossa analis Westwood, 1840 é sugerida como possível indicadora de florestas mais preservadas. Na comparação entre as cinco áreas foram verificadas correlações positivas e significativas da riqueza de espécies de abelhas com o tamanho da área e da diversidade de abelhas $\left(\mathrm{H}^{\prime}\right)$ com a diversidade florística $\left(\mathrm{H}^{\prime}\right)$. Estes dados sugerem que perdas de área e qualidade de hábitat influenciam negativamente a comunidade destas abelhas, reduzindo a riqueza e diversidade de espécies. Os maiores valores de similaridade foram observados na comparação entre os fragmentos da região do Imbaú, distantes entre si por até $2 \mathrm{Km}$, sugerindo que estes não estejam isolados para as populações de Euglossina, ou que venham sofrendo igualmente os efeitos da fragmentação.
\end{abstract}

PALAVRAS-CHAVE. Abelhas de orquídeas; fragmentação florestal; bioindicadores; Mata Atlântica.

A Mata Atlântica, considerada um dos principais "hotspots" para conservação (Myers et al. 2000) teve no estado do Rio de Janeiro cerca de $84 \%$ da sua cobertura florestal original retirada (Fundação SOS Mata Atlântica/INPE 2001). O que restou desta cobertura encontra-se dividida em diversos fragmentos de tamanhos variados, estando a maior parte em regiões montanhosas e uns poucos em áreas submontanas, margens de rios e ecossistemas litorâneos (Rocha et al. 2003). Outro problema é o pequeno tamanho dos fragmentos, o que pode ser insuficiente para manter populações animais sustentáveis, tanto no aspecto ecológico como no genético (Lande, 1988). Neste sentido, têm-se buscado atualmente espécies animais e vegetais que possam ajudar a compreender melhor os efeitos da fragmentação. Entre estas espécies, as abelhas da subtribo Euglossina (Hymenoptera; Apidae; Apini) têm sido indicadas por suas características como facilidade de captura, de identificação taxonômica e abundância ao longo do ano (Powell \& Powell 1987; Peruquetti et al. 1999).

As abelhas Euglossina estão distribuídas principalmente na região neotropical, do México até a Argentina (Dodson et al. 1969; Dressler 1982). Elas estão entre as principais espécies polinizadoras nas florestas tropicais e subtropicais nas Américas Central e do Sul. Roubik \& Hanson (2004) citam 68 famílias de plantas utilizadas para a obtenção de néctar e pólen e quatro para resina. Uma das principais características destas abelhas é a relação dos machos com espécies da família Orchidaceae e outras fontes de compostos aromáticos (Dodson \& Frymire 1961; Dressler 1982, 1990, 1993; Roubik 1989; Singer 
2003), por razões ainda não totalmente esclarecidas. Uma técnica que tem sido empregada e que permitiu um grande avanço no estudo desta subtribo, principalmente em áreas florestais, é a utilização de fragrâncias sintetizadas para atração de machos, para posterior captura e identificação (Powell \& Powell 1987; Becker et al. 1991; Rebêlo \& Garófalo 1991, 1997; Peruquetti et al. 1999; Tonhasca et al. 2002).

A importância de se preservar o que ainda resta da Mata Atlântica, aliada à ausência de dados sobre seus polinizadores na região da bacia do Rio São João, considerada uma das mais importantes em termos de áreas remanescentes florestais do estado do Rio de Janeiro (Carvalho et al. 2006), torna de extrema relevância estudos sobre a ocorrência e a diversidade deste grupo. Assim, os objetivos deste trabalho foram: (1) analisar a riqueza e composição de espécies, abundância e sazonalidade de abelhas Euglossina em remanescentes de Floresta Atlântica submontana na bacia do Rio São João, com diferentes níveis de alteração antrópica, (2) comparar os resultados obtidos em cada remanescente, visando relacionar os efeitos de duas variáveis do habitat (diversidade florística e tamanho da área) sobre a diversidade de Euglossina.

\section{MATERIALE MÉTODOS}

Áreas de Estudo. As áreas de estudo utilizadas são remanescentes de Mata Atlântica no estado do Rio de Janeiro, localizados na região da bacia do rio São João (Fig.1), cuja área de $3000 \mathrm{~km}^{2}$ abrange oito municípios: Araruama, Cabo Frio, Cachoeiras do Macacu, Casimiro de Abreu, Rio Bonito, Rio das Ostras, São Pedro da Aldeia e Silva Jardim. Nessa região, a vegetação é caracterizada como Floresta Ombrófila Densa de baixada e submontana (Rodrigues 2004; Carvalho et al. 2006). As chuvas ocorrem com médias anuais em torno de $1500 \mathrm{~mm}$, ocorrendo um período seco que pode chegar a 60 dias (Rocha et al. 2003).

As cinco áreas selecionadas foram: Reserva Biológica União com 3126 ha $\left(22^{\circ} 26^{\prime} \mathrm{S} 42^{\circ} 02^{\prime} \mathrm{W}\right)$, e quatro fragmentos florestais situados na região do rio Imbaú: Andorinhas com 145 ha ( $\left.22^{\circ} 37^{\prime} \mathrm{S} 42^{\circ} 26^{\prime} \mathrm{W}\right)$, Imbaú com 130 ha ( $22^{\circ} 36^{\prime} \mathrm{S} 42^{\circ} 28^{\prime} \mathrm{W}$ ), Afetiva com 19 ha $\left(22^{\circ} 38^{\prime} \mathrm{S} 42^{\circ} 27^{\prime} \mathrm{W}\right)$ e Estreito com 21 ha $\left(22^{\circ} 37^{\prime} \mathrm{S} 42^{\circ} 27^{\prime} \mathrm{W}\right)$. Segundo relatos de antigos moradores, os fragmentos da região do Imbaú formavam um contínuo florestal, todos com históricos de fragmentação variando a partir das décadas de 1950-1970 e que atualmente se encontram com diferentes tamanhos e em diferentes condições.

Metodologia. Sete iscas aromáticas já utilizadas tradicionalmente em outros estudos foram utilizadas neste trabalho: cineol, eugenol, eucaliptol, vanilina, acetato de benzila, salicilato de metila e cinamato de metila. Chumaços de algodão embebidos com estas iscas foram colocados nas armadilhas (uma isca por armadilha) desenvolvidas conforme o modelo apresentado na Fig. 2.

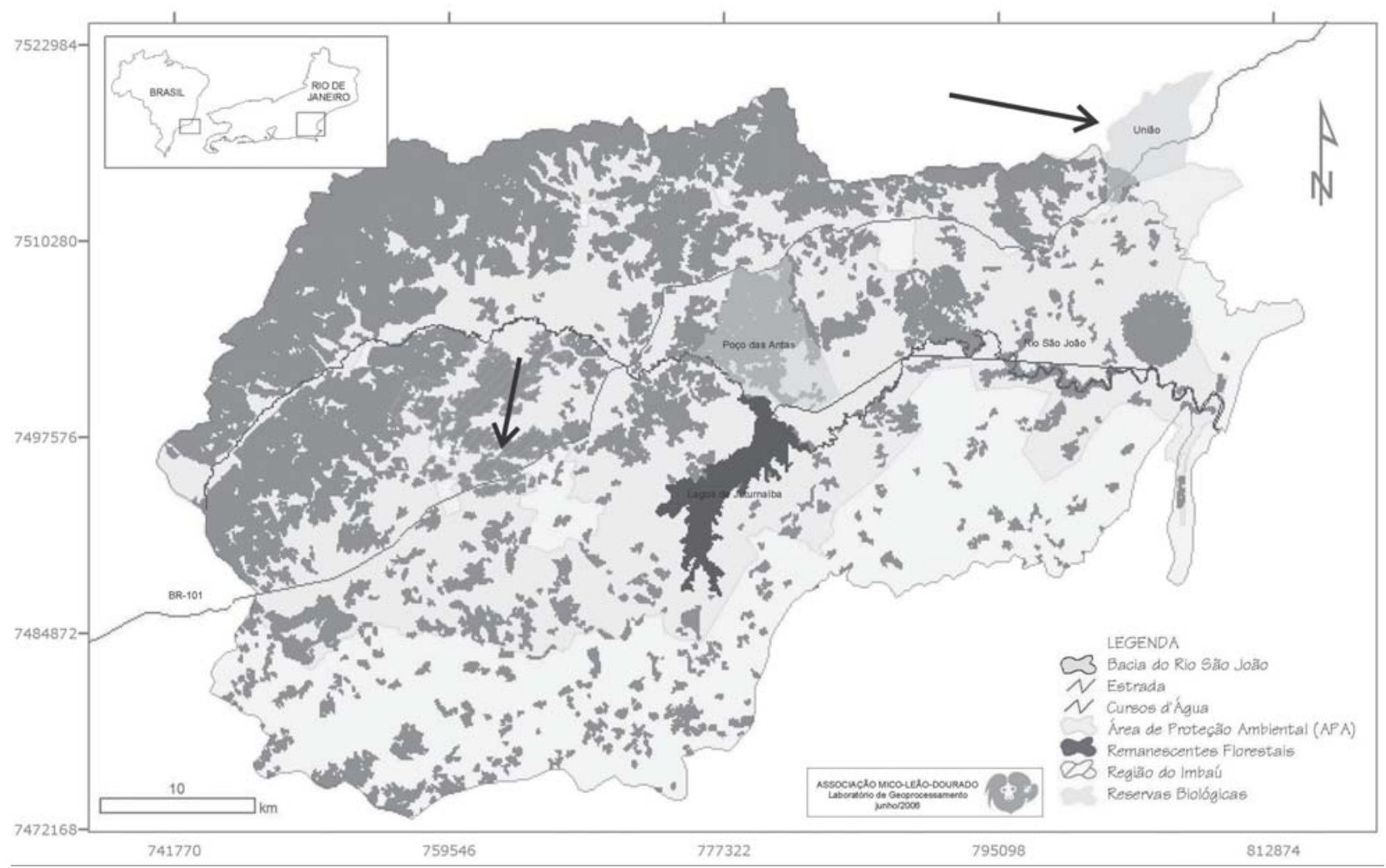

Fig. 1. Localização dos fragmentos de estudo na Bacia do rio São João, situada nos municípios de Casimiro de Abreu e Silva Jardim, RJ. Setas indicam a região do Imbaú e a Reserva Biológica União. Fonte: imagem obtida por LandSat e editada pelo setor de geoprocessamento da Associação Mico -Leão Dourado. 


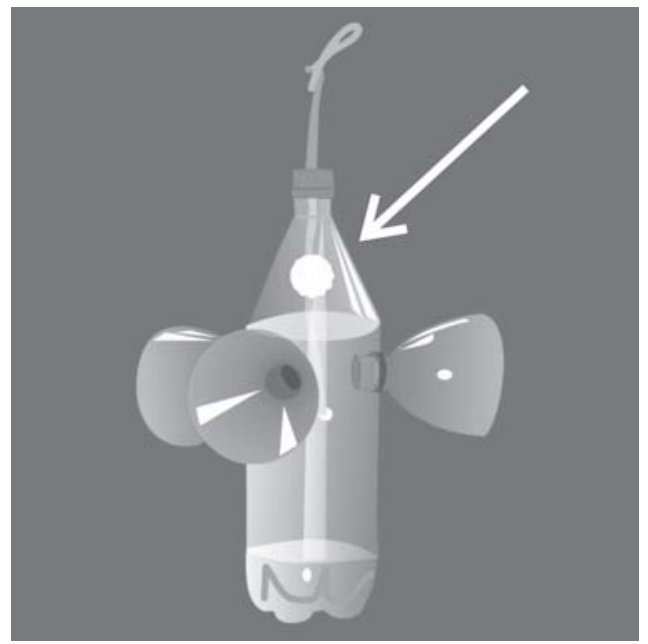

Fig. 2. Armadilha confeccionada a partir de garrafas pet que foi instalada nas estações de coleta nas áreas selecionadas, R.B.União em Casimiro de Abreu (RJ) e nos fragmentos da região do Imbaú em Silva Jardim (RJ). A seta indica o local onde está situado o algodão contendo a isca aromática.

As armadilhas foram instaladas às $8 \mathrm{~h}$, a uma altura de $1,5 \mathrm{~m}$ e separadas entre si por uma distância de $2 \mathrm{~m}$, e os indivíduos foram coletados às $15 \mathrm{~h}$. A captura na R.B. União foi feita em um único dia, com a instalação simultânea em três pontos. Em dias subseqüentes, as armadilhas foram instaladas em um fragmento pequeno (Estreito ou Afetiva, 1 ponto) e um médio (Andorinhas ou Imbaú, dois pontos), a cada dia, requerendo assim três dias por mês para a captura em todas as áreas. Em cada ponto, foi instalado um conjunto de sete armadilhas, cada uma contendo uma isca aromática. As coletas foram feitas uma vez por mês, no período entre novembro de 2004 e outubro de 2005, em dias com boa estabilidade climática, sem ameaça de chuva. Os espécimes capturados foram depositados na Coleção de Zoologia do Laboratório de Ciências Ambientais na Universidade Estadual do Norte Fluminense.

Nos fragmentos pequenos o ponto de amostragem foi colocado na área central visando cobrir todo o fragmento com o raio de ação das armadilhas. A instalação de maior número de pontos de amostragem nestes fragmentos não pareceu viável, devido ao pequeno tamanho dos fragmentos. Nos fragmentos médios e na R. B. União tentou-se cobrir o máximo de área possível mantendo sempre uma distância maior que 300 m entre um grupo de armadilhas e outro, a fim de evitar a sobreposição do raio de ação das armadilhas. Na R. B.União, assim como nos fragmentos médios, os pontos não foram fixos pois as coletas foram realizadas visando abranger a maior heterogeneidade de habitat possível.

Os dados climáticos (precipitação e temperatura média) foram obtidos na Reserva Biológica de Poço das Antas, ponto central na Bacia do Rio São João, por integrantes do Jardim Botânico do Rio de Janeiro envolvidos no Projeto Mata Atlântica.

Análise dos Dados. A média de abundância entre os pontos no mesmo fragmento (abundância média), ou densidade de abelhas atraídas a um conjunto de armadilhas, foi utilizada para comparar a diferença na abundância de indivíduos em cada área.

O índice de diversidade utilizado foi o de Shannon (Magurran 2003): $H^{\prime}=-\Sigma \mathrm{p}_{\mathrm{i}} \ln \mathrm{p}_{\mathrm{i}}$, onde $\mathrm{p}_{\mathrm{i}}=$ proporção de indivíduos representados na amostra pela espécie $\mathrm{i}, \ln =$ logaritmo neperiano. Este foi calculado com o auxílio do software EcoSim 7 (Gotelli e Entsminger 2001). Este mesmo software foi utilizado para realizar as correlações segundo modelos nulos, entre: tamanho da área e abundância média de abelhas; tamanho da área e riqueza de espécies de abelhas; diversidade arbórea e diversidade de espécies de abelhas. A dominância de espécies em cada área foi obtida pelo índice de Berger-Parker (Magurran 2003), obtido por: $d=\mathrm{N}_{\text {máx }} / \mathrm{N}$, onde $\mathrm{d}$ = dominância, $\mathrm{N}_{\text {máx }}=\mathrm{o}$ número de indivíduos da espécie mais abundante, $\mathrm{N}=$ número total de indivíduos amostrados na área. Para obter-se uma estimativa da diversidade e equidade das espécies foi utilizado o Rank-Abundance Plot (Whittaker 1965) e a diferença entre as curvas obtidas para as diferentes áreas foi analisada pelo teste de Kolmogorov-Smirnov (Magurran 2003). A similaridade entre as áreas foi analisada pelo índice de similaridade de Renkonen recomendado por Wolda (1981) para pequenas amostras.

\section{RESULTADOS}

Foi registrado um total de 4094 machos de 17 espécies de Euglossina ao longo de um ano, entre novembro de 2004 e outubro de 2005, nas cinco áreas amostradas (Tab. I).

A abundância média por pontos (Tab. I), na R. B. União (641) foi a maior observada entre as áreas estudadas. Apesar do fragmento Andorinhas (261) apresentar abundância média menor que os fragmentos pequenos Estreito (287) e Afetiva (433), a comparação entre densidade de indivíduos atraídos a um conjunto de armadilhas e tamanho da área, incluindo todas as áreas estudadas, mostrou uma relação positiva entre abundância média e tamanho das áreas $(\mathrm{r}=0,80 ; \mathrm{p}=0,1)$, embora não significativa. Os indivíduos capturados em todos os fragmentos estudados representaram 17 espécies

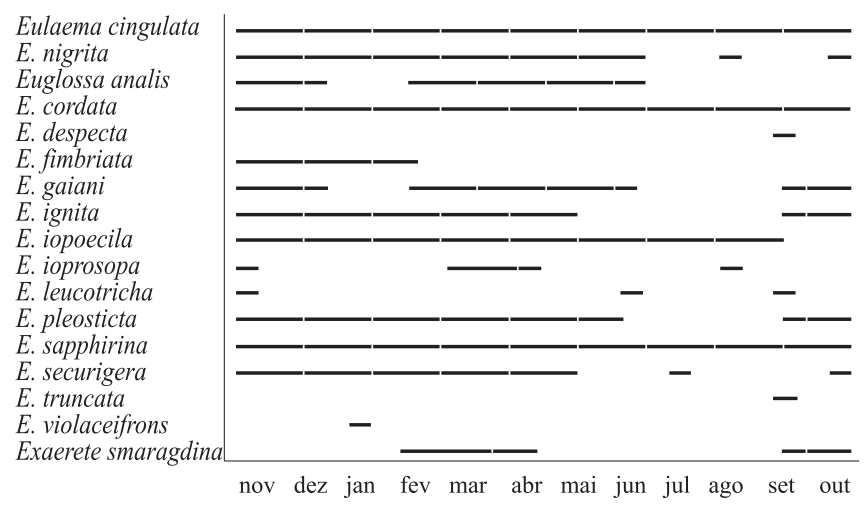

Fig. 3. Distribuição das 17 espécies de abelhas Euglossina capturadas ao longo de 12 meses nos fragmentos da região do Imbaú (Silva Jardim, RJ) e R.B.União (Casimiro de Abreu, RJ). 
Tabela I. Composição, abundância, riqueza, dominância e iscas visitadas por espécies de Euglossina entre novembro de 2004 e outubro de 2005 na Reserva Biológica União (R.B. União, Casimiro de Abreu, RJ) e nos fragmentos da região do Imbaú: AFT=Afetiva, EST=Estreito, IMB=Imbaú e AND=Andorinhas (Silva Jardim, RJ). As iscas foram numeradas como: (1) Eugenol; (2) Cinamato de Etila; (3) Vanilina; (4) Cineol; (5) Acetato de Benzila; (6) Salicilato de Metila; e (7) Eucaliptol.

\begin{tabular}{|c|c|c|c|c|c|c|c|c|c|c|c|c|}
\hline \multirow[t]{2}{*}{ Espécies } & \multicolumn{6}{|c|}{ Abundância por área } & \multicolumn{5}{|c|}{ Abundância Relativa } & \multirow[t]{2}{*}{ Iscas } \\
\hline & AFT & EST & IMB & AND & R.B.União & Total & AFT & EST & IMB & AND & R.B.União & \\
\hline Eulaema cingulata (Fabricius, 1804) & 56 & 45 & 69 & 43 & 251 & 464 & 12,9 & 15,7 & 7,4 & 8,2 & 13,1 & $1,3,5,7$ \\
\hline Eulaema nigrita Lepeletier, 1841 & 34 & 27 & 22 & 42 & 165 & 290 & 7,9 & 9,4 & 2,4 & 8 & 8,6 & $3,4,7$ \\
\hline Euglossa analis Westwood, 1840 & - & - & - & - & 169 & 169 & - & - & - & - & 8,8 & $1,4,7$ \\
\hline Euglossa cordata (Linnaeus, 1758) & 260 & 175 & 721 & 377 & 1118 & 2651 & 60 & 61 & 77,6 & 72,2 & 58,2 & $1,2,4,6,7$ \\
\hline Euglossa despecta Moure, 1968 & - & - & - & - & 1 & 1 & - & - & - & - & 0,1 & 4 \\
\hline Euglossa fimbriata Moure, 1968 & 1 & - & - & 1 & 8 & 10 & 0,2 & - & - & 0,2 & 0,4 & $2,4,7$ \\
\hline Euglossa gaianii Dressler, 1982 & 2 & 1 & 4 & - & 15 & 22 & 0,5 & 0,3 & 0,4 & - & 0,8 & 2,6 \\
\hline Euglossa ignita Smith, 1874 & - & - & - & - & 39 & 39 & - & - & - & - & 2,0 & $2,4,5,6$ \\
\hline Euglossa iopoecila Dressler, 1982 & - & - & 2 & - & 67 & 69 & - & - & 0,2 & - & 3,5 & $1,4,7$ \\
\hline Euglossa ioprosopa Dressler, 1982 & - & - & 4 & - & 1 & 5 & - & - & 0,4 & - & 0,1 & $2,4,7$ \\
\hline Euglossa leucotricha Rebêlo \& Moure, 1996 & - & 1 & 1 & - & 1 & 3 & - & 0,3 & 0,1 & - & 0,1 & 2 \\
\hline Euglossa pleosticta Dressler, 1982 & 19 & 6 & 23 & 13 & 15 & 76 & 4,4 & 2,1 & 2,5 & 2,5 & 0,8 & $1,4,3,6,7$ \\
\hline Euglossa sapphirina Moure, 1968 & 51 & 30 & 72 & 33 & 51 & 237 & 11,8 & 10,5 & 7,8 & 6,3 & 2,7 & $3,4,7$ \\
\hline Euglossa securigera Dressler, 1982 & 5 & 2 & 10 & 4 & 18 & 39 & 1,2 & 0,7 & 1,1 & 0,8 & 0,9 & $1,2,4,7$ \\
\hline Euglossa truncata Rebêlo \& Moure, 1996 & - & - & - & - & 1 & 1 & - & - & - & - & 0,1 & 1 \\
\hline Euglossa violaceifrons Rebêlo \& Moure, 1996 & - & - & - & - & 2 & 2 & - & - & - & - & 0,1 & 4 \\
\hline Exaerete smaragdina (Guérin, 1844) & 5 & - & 1 & 9 & 1 & 16 & 1,2 & - & 0,1 & 1,7 & 0,1 & $2,3,7$ \\
\hline Abundância: Total & 433 & 287 & 929 & 522 & 1923 & 4094 & & & & & & \\
\hline Média & 433 & 287 & 464,5 & 261 & 641 & & & & & & & \\
\hline Riqueza & 9 & 8 & 11 & 8 & 17 & & & & & & & \\
\hline Dominância & 60 & 61 & 77,6 & 72,2 & 58,2 & & & & & & & \\
\hline
\end{tabular}

pertencentes a três gêneros: Euglossa, Eulaema e Exaerete (Tab. I). As espécies mais generalistas em termos de visita a diferentes iscas foram Euglossa cordata (Linnaeus 1758) e Euglossa pleosticta Dressler, 1982, seguidas por Eulaema cingulata (Fabricius 1804), Euglossa ignita (Smith 1874) e Euglossa securigera Dressler, 1982 (Tab. I).

A R. B. União foi a área que apresentou o maior número de espécies (17, sendo 5 exclusivas). Entre os fragmentos, Imbaú (130 ha) deteve o maior número de espécies (11), seguido em ordem decrescente por Afetiva (19 ha e 9spp), Andorinhas ( 145 ha e $8 \mathrm{spp}$ ) e Estreito (21 ha e $8 \mathrm{spp}$ ). A riqueza de espécies de Euglossina mostrou uma relação positiva e significativa com o tamanho da área $(\mathrm{r}=0,95 ; \mathrm{p}=0,01)$.

A dominância das espécies foi menor na R. B. União em relação aos fragmentos do Imbaú (Tab. I). Entre estes fragmentos, Afetiva foi o que apresentou a menor dominância seguido por Estreito, Andorinhas e Imbaú, respectivamente. Euglossa cordata apresentou os maiores valores de abundância relativa e Eulaema cingulata foi uma das espécies mais abundantes em todas as áreas, com ocorrência ao longo de todo o ano (Fig. 3). Euglossa sapphirina Moure, 1968 também ocorreu ao longo do ano, com alta abundância relativa nos fragmentos do Imbaú, enquanto que Euglossa analis Westwood, 1840 ocorreu exclusivamente na R. B. União, onde foi a terceira espécie mais abundante.

A abundância de indivíduos variou ao longo do ano (Fig. 4 ), sendo os menores valores observados entre os meses de junho e setembro, com queda acentuada em julho. Um pico na abundância total de abelhas foi observado em fevereiro em todas as áreas, com grande contribuição de Euglossa cordata. O número de espécies ocorrendo nos cinco fragmentos foi maior nos meses de novembro (13), e de fevereiro a abril (12 em cada mês), enquanto que os menores números ocorreram em julho (5) e agosto (6).

A diversidade de Euglossina calculada pelo índice de Shannon (Tab. II) foi maior na R. B. União, seguida pelos fragmentos pequenos (Afetiva e Estreito) e pelos médios (Andorinhas e Imbaú). As curvas obtidas para importância das espécies (Rank-Abundance Plot) não apresentaram diferença significativa na comparação entre todos os

Tabela II. Diversidade Florística dos fragmentos florestais (índice de Shannon, H'), segundo Carvalho et al., 2006, com base em levantamento das espécies vegetais arbóreas DAP $\geq 10$ e diversidade de machos de Euglossina, atraídos a iscas aromáticas entre novembro de 2004 e outubro de 2005 .

\begin{tabular}{lccc}
\hline Fragmento & $\begin{array}{c}\text { Área } \\
\text { amostral }\end{array}$ & $\begin{array}{c}\text { Diversidade } \\
\text { florística (H') }\end{array}$ & $\begin{array}{c}\text { Diversidade de } \\
\text { Euglossina (H') }\end{array}$ \\
\hline Afetiva (19 ha) & 0,2 ha & 3,33 & 1,3 \\
Estreito (21 ha) & 0,2 ha & 2,11 & 1,2 \\
Imbaú (130 ha) & 0,2 ha & 3,06 & 0,89 \\
Andorinhas (145 ha) & 0,2 ha & 3,34 & 1,02 \\
R.B.União (2300 ha)* & 0,4 ha & 4,38 & 1,47 \\
\hline
\end{tabular}

*dados referentes à mata madura 

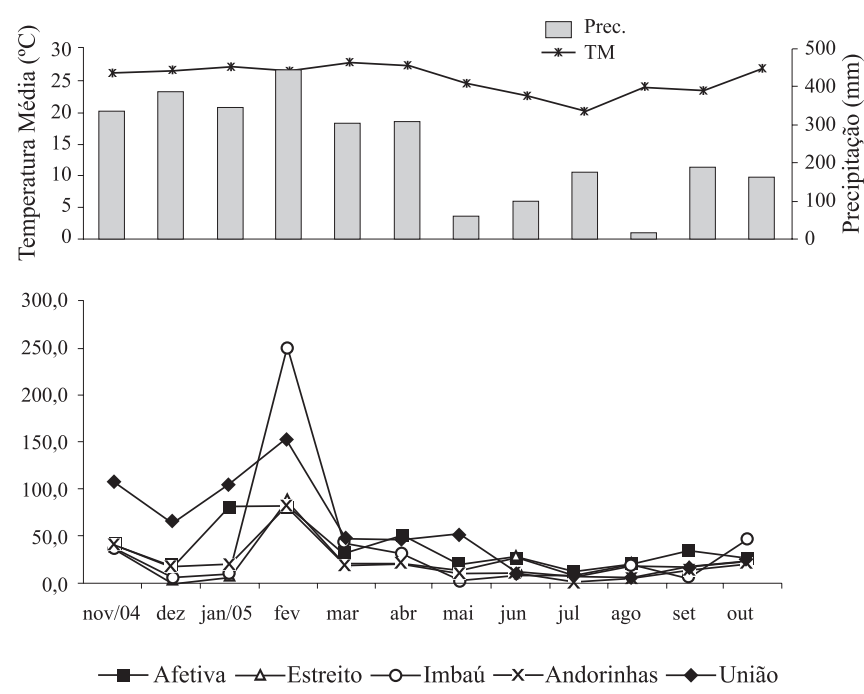

Fig. 4. Parâmetros climáticos (temperatura média e precipitação) e abundância média por ponto de amostragem de Euglossina por mês ao longo de 12 meses de coleta nos quatro fragmentos da região do Imbaú (Silva Jardim, RJ) e na R.B.União (Casimiro de Abreu, RJ).

fragmentos (segundo o teste de Kolmogorov-Smirnov, p > 0,05) (Fig. 5).

A correlação entre diversidade $\left(\mathrm{H}^{\prime}\right)$ de Euglossina e diversidade $\left(\mathrm{H}^{\prime}\right)$ florística foi positiva e estatisticamente significativa $(r=0,99 ; p=0,009)$.

A similaridade percentual de Renkonen para as áreas amostradas foi alta, sendo $81 \%$ o valor mais baixo obtido na comparação entre os fragmentos da região do Imbaú (Tab. III). Os fragmentos pequenos (Afetiva e Estreito) foram muito semelhantes entre si $(94,4 \%)$, assim como os fragmentos médios (Andorinhas e Imbaú, 91,6\%). Os menores valores de similaridade foram encontrados na comparação entre R. B. União e os fragmentos de tamanho médio.

\section{DISCUSSÃO}

O número de espécies amostrado neste estudo foi menor do que o encontrado por Tonhasca et al. (2002) em fragmentos de Mata Atlântica submontana na região do Parque Estadual do Desengano, também no norte do Estado do Rio de Janeiro (21 espécies), cujo estudo foi realizado durante 3 anos. Cinco espécies amostradas na bacia do Rio São João (Euglossa despecta Moure, 1968; E. violaceifrons Rebêlo \& Moure, 1996; E. leucotricha Rebêlo \& Moure, 1995; E. ioprosopa Dressler, 1982; e E. gaianii Dressler, 1982) não foram encontradas por Tonhasca (op.cit), enquanto que 9 espécies citadas para o $\mathrm{P}$. E. Desengano, não foram encontradas nas nossas amostragens. Ainda na comparação entre estas duas áreas, $67 \%$ das espécies coletadas na bacia do rio São João foram também amostradas por Tonhasca et al. (2002), indicando similaridade na composição entre as áreas. Além do período de estudo, outros fatores como diferentes metodologias de amostragem ou diferenças regionais de habitat, tornam de difícil interpretação a comparação entre estes estudos. Entretanto, o número de espécies encontrado neste trabalho representa uma riqueza significativa. Estudos realizados em outras áreas de mata atlântica, revelaram números menores na Paraíba (11 espécies, Souza et al. 2005), Bahia (5 espécies, Raw 1989) e em floresta estacional em Minas Gerais (7 espécies, Nemésio 2003) e no Paraná (7 espécies, Sofia \& Suzuki 2004).

A abundância de Euglossa cordata e Euglossa sapphirina influenciou fortemente os picos de abundância de Euglossina em fevereiro, observados em todas as áreas. Em ano anterior, durante amostragens em um projeto piloto, foi observado um pico de abundância de Eulaema cingulata no mês de setembro; entretanto, este padrão não se repetiu no ano de estudo. Os dados de sazonalidade da comunidade de Euglossina foram semelhantes aos encontrados por outros autores, cujos estudos também verificaram maior número de indivíduos na estação chuvosa em relação à estação seca (Becker et al. 1991; Rebêlo \& Garófalo 1991; Oliveira \& Campos 1995; Oliveira 1999). A explicação para este padrão de distribuição pode estar associada a diversos fatores como picos de floração (Pearson \& Dressler 1985) ou atividades de nidificação e emergência de adultos (Ackerman 1983; Roubik \& Ackerman 1987; Rebêlo \& Garófalo 1991).

Diversos estudos realizados ao longo do Brasil têm apresentado resultados distintos com relação aos efeitos da fragmentação sobre a diversidade das abelhas da subtribo Euglossina. Enquanto trabalhos como o de Powell \& Powell (1987) indicaram um declínio populacional relacionado à menor área de fragmento, outros trabalhos como Becker et al. (1991) e Tonhasca et al. (2002) não verificaram esta relação. Sofia \& Suzuki (2004) observaram uma relação entre tamanho da área e abundância quando comparado um fragmento de 2280 ha com os de 86 ha e 8,5 ha, não evidenciando, porém, esta relação quando comparados os dois menores fragmentos na Mata Atlântica do Paraná. Nosso trabalho sugere que a riqueza de espécies de Euglossina está relacionada ao tamanho do fragmento florestal na bacia do Rio São João. Entretanto, este não deve ser o único fator determinante. A maior riqueza e diversidade na R. B. União é provavelmente devida ao grande tamanho aliado à maior proteção da área que leva à melhor qualidade ambiental e diversidade florística. Esta relação de maior diversidade de Euglossina em fragmentos florestais com maior diversidade florística também foi observada no nosso

Tabela III. Similaridade (Renkonnen) entre os quatro fragmentos da região do Imbaú (Silva Jardim, RJ) e a R.B.União (Casimiro de Abreu, RJ) quanto à composição de espécies de abelhas Euglossina atraídas a iscas aromáticas ao longo de um ano de coleta (novembro 2004 a outubro 2005). Os valores encontram-se em porcentagem.

Similaridade (Renkonnen) entre as áreas estudadas

\begin{tabular}{lccccc}
\hline & Afetiva & Estreito & Imbaú & Andorinhas & R.B.União \\
Afetiva & 1 & 94,4 & 81,7 & 87 & 84 \\
Estreito & & 1 & 81 & 86 & 84,3 \\
Imbaú & & & 1 & 91,6 & 73,1 \\
Andorinhas & & & & 1 & 78 \\
R.B.União & & & & & 1 \\
\hline
\end{tabular}




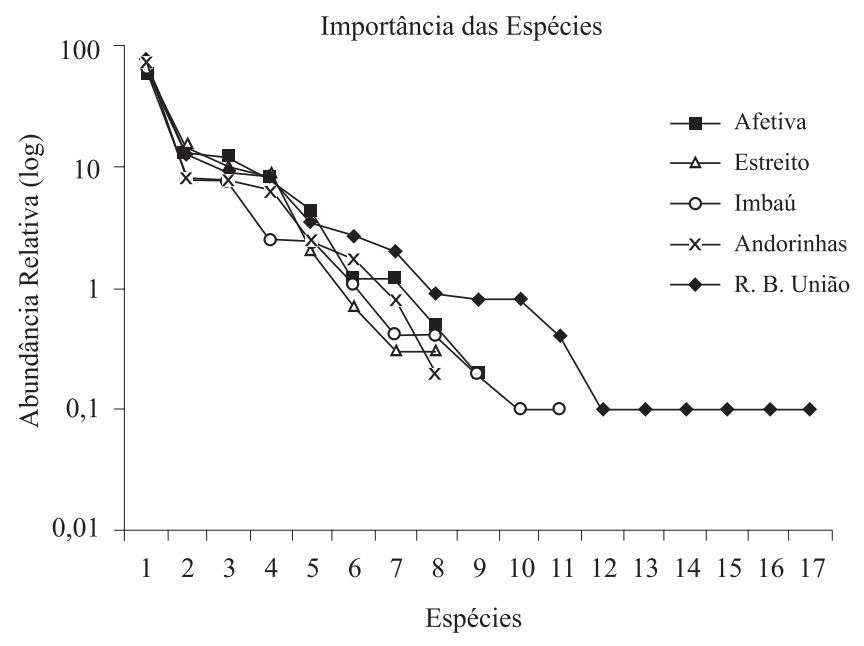

Fig. 5. Importância das espécies (Rank-abundance plot, Whittaker 1965) de acordo com sua abundância relativa (tabela I) em escala logarítmica, de Euglossina atraídas a iscas aromáticas entre novembro de 2004 e outubro de 2005 nos quatro fragmentos da região do Imbaú (Silva Jardim, RJ) e na R.B.União (Casimiro de Abreu, RJ).

estudo. Outros autores, como Peruquetti et al. (1999) mostraram que fragmentos maiores e menos perturbados podem apresentar maior riqueza de espécies deste grupo de abelhas. Outro fator a ser considerado importante na determinação da riqueza de espécies de uma área é a heterogeneidade ambiental, certamente muito maior na R. B. União do que em outros fragmentos estudados. Já na comparação entre os fragmentos estudados menores do que 150 ha, o estado de conservação e a diversidade florística podem ser mais importantes na comparação de riqueza de espécies de Euglossina do que o tamanho do fragmento.

A matriz do entorno tem sido discutida como um dos fatores determinantes na composição de espécies do fragmento. A proximidade entre os fragmentos na região do Imbaú deve favorecer o deslocamento destas abelhas entre fragmentos, apesar da matriz desfavorável composta predominantemente por pastagem, o que poderia explicar a grande similaridade na composição de espécies observada entre estes fragmentos.

A distância e as barreiras (naturais ou não) entre fragmentos florestais têm sido consideradas de extrema importância em estudos que visam compreender os efeitos da degradação do ambiente sobre comunidades, inclusive de abelhas. A capacidade de dispersão das abelhas Euglossina e de exploração de áreas próximas poderia explicar a composição semelhante entre os fragmentos do Imbaú, de forma similar ao discutido por Tonhasca et al. $(2002,2003)$ para a região do Imbé no norte do Estado do Rio de Janeiro. Experimentos preliminares de marcação e recaptura entre os fragmentos do Imbaú corroboram esta hipótese (Ramalho, observação pessoal). Além disso, não se deve esquecer o fato de que estas áreas formaram no passado um contínuo florestal, e neste caso, é possível que a grande similaridade na composição entre as áreas seja resultado de semelhantes efeitos acarretados pelas perdas de área e degradação nestes fragmentos menores do que 150 ha.
A distribuição do número de indivíduos entre as espécies assemelha-se aos resultados obtidos em diversos outros trabalhos (Ricklefs et al. 1969; Janzen et al. 1982; Ackerman 1983; Oliveira \& Campos 1995; Rebêlo \& Garófalo 1997; Sofia \& Suzuki 2004), nos quais poucas espécies são representadas por muitos indivíduos e muitas espécies representadas por poucos indivíduos. Embora ocorram diferenças de abundância entre as áreas estudadas, estas não são estatisticamente significativas quando analisadas dentro de um ranking de espécies mais abundantes.

Os fragmentos da região do Imbaú apresentaram uma grande similaridade quanto às espécies de maior abundância relativa, porém ocorreram pequenas diferenças na composição de espécies raras. Esta diferença na composição pode ser devida à maior concentração de espécies em áreas com maior disponibilidade de recursos de interesse (Armbruster 1993). Entretanto, deve-se considerar que o pequeno número de fragmentos estudados e o curto período de amostragem podem estar influenciando neste resultado, principalmente tratandose de espécies raras.

Entre as espécies amostradas destacam-se Euglossa cordata, Eulaema nigrita e Euglossa analis, espécies consideradas em outros estudos como possíveis espécies indicadoras. Trabalhos recentes vêm sugerindo que Euglossa cordata e Eulaema nigrita sejam espécies favorecidas por ambientes secos e alterados, podendo ser consideradas indicadoras de ambientes degradados (Rebêlo \& Cabral 1997; Peruquetti et al. 1999; Rebêlo \& Silva 1999; Silva \& Rebêlo 2002; Tonhasca et al. 2002). Nossos dados mostram que estas espécies podem ocorrer em grande número em áreas com diferentes tamanhos e condições de conservação, não corroborando portanto a sua ocorrência como um parâmetro indicador de área degradada na região da bacia do rio São João. Por outro lado, sugerimos que Euglossa analis possa ser uma espécie indicadora de fragmentos de boa qualidade, já que só foi evidenciada na área mais preservada (R. B. União), onde ocorreu como a terceira espécie mais abundante. Esta sugestão havia sido indicada por outros autores, em outros estudos de comunidades de Euglossina em Mata Atlântica (Bonilla Gómez 1999; Tonhasca et al. 2002).

Apesar dos parâmetros individuais (abundância média e riqueza) dos fragmentos da região do Imbaú serem menores que os da R. B. União quando tratados separadamente, a região como um todo detém uma diversidade quase tão alta quanto a R. B. União, sugerindo que toda a área deva ser protegida para a manutenção das populações de Euglossina, não sendo indicada a proteção de somente algumas áreas prioritárias dentro desta paisagem. É importante que os estudos não vejam as áreas como isoladas, fora de um contexto de paisagem, pois espécies com capacidade de dispersão podem utilizá-las como um todo, sendo a conservação de toda a região importante para sua manutenção, principalmente tratando-se de um ecossistema tão devastado como a Mata Atlântica.

Agradecimentos. Aos amigos e colaboradores: Willian Aguiar, Anselma Reis, Heuzenil Cordeiro, Helmo Carvalho, Carlos Pereira Júnior 
e Leonardo Resende pela ajuda no trabalho de campo. Aos doutores: Carlos R.Ruiz Miranda, Leandro R. Monteiro e Gilberto S. Albuquerque pela colaboração no desenvolvimento do projeto. Ao IBAMA / Instituto Chico Mendes / R. B. União e à Associação Mico Leão Dourado pelo apoio, logística e pela confecção do mapa apresentado na figura 1. Aos revisores anônimos pelas sugestões feitas ao trabalho. A Faperj (bolsa de mestrado ao primeiro autor) e CEPF (Critical Ecosystems Partnership Fund) pelo financiamento. Esta é uma contribuição do Programa de Pós-Graduação em Ecologia e Recursos Naturais da UENF.

\section{REFERÊNCIAS}

Ackerman, J. D. 1983. Specificity and mutual dependency of the orchideuglossine bee interaction. Biological Journal of the Linnean Society 20: $301-314$.

Armbruster, W. S. 1993. Within-habitat heterogeneity in baiting samples of male euglossine bees: possible causes and implications. Biotropica 25: 122-128.

Becker, P.; J. S. Moure \& F. J. A. Peralta. 1991. More about Euglossine bees in Amazonian forest fragments. Biotropica 23: 586-591.

Bonilla-Gómez, M. A. 1999. Caracterização da estrutura espaçotemporal da comunidade de abelhas euglossinas (Hymenoptera, Apidae) na Hiléia Baiana. Tese de Doutorado. Universidade Estadual de Campinas, $153 \mathrm{p}$.

Carvalho, F. A.; M. T. Nascimento \& J. M. A. Braga. 2006. Composição e riqueza florística do componente arbóreo da Floresta Atlântica submontana na região de Imbaú, município de Silva Jardim, RJ. Acta Botanica Brasilica 20: $727-740$.

Dodson, C. H. \& G. P. Frymire. 1961. Natural pollination of orchid. Missouri Botanical Garden Bulletin 49: 133-152.

Dodson, C. H.; R. L. Dressler; H. G. Hills; R. M. Adams \& N. H. Williams. 1969. Biologically active compounds in orchid fragrances. Science 164: 1243-1249.

Dressler, R. L. 1982. Biology of the orchid bees (Euglossini). Annual Review of Ecology and Systematics 13: 373-394.

Dressler, R. L. 1990. The orchids, natural history and classification. Harvard University Press, Cambridge, 332 p.

Dressler, R. L. 1993. Phylogeny and classification of the orchid family. Dioscorides Press, Portland, Oregon, $314 \mathrm{p}$.

Fundação SOS Mata Atlântica/INPE. 2001. Atlas dos remanescentes florestais do Rio de Janeiro.

Gotelli, N. J. \& G. L. Entsminger. 2001. EcoSim: Null models software for ecology. Version 7.0. Acquired Intelligence Inc. \& Kesey-Bear.

Janzen, D. H.; P. J. de Vries; M. L. Higgins \& L. S. Kimsey. 1982. Seasonal and site variation in Costa Rican euglossine bees at chemical baits in lowland deciduous and evergreen forests. Ecology 63: 674.

Lande, R. 1988. Genetics and demography in biological conservation. Science 241: 1455-1460.

Magurran, A. E. 2003. Measuring Biological Diversity. Blackwell Publishing, Oxford, $256 \mathrm{p}$.

Myers, N.; R. A. Mittermeier; C. G. Mittermeier; G. A. B. Fonseca \& J. Kent . 2000. Biodiversity hotspots for conservation priorities. Nature 403: 853-858.

Nemésio, A. 2003. Preliminary sampling of Euglossina (Hymenoptera: Apidae: Apini) of Reserva Particular do Patrimônio Natural "Feliciano Miguel Abdala", Caratinga, Minas Gerais, southeastern Brazil. Lundiana 4: 121-124

Oliveira, M. L. 1999. Sazonalidade e horário de atividade de abelhas euglossinae (Hymenoptera, Apidae), em florestas de terra firme na Amazônia central. Revista Brasileira de Zoologia 16: 83-90.

Oliveira, M. L. \& L. A. O. Campos. 1995. Abundância, riqueza e diversidade de abelhas euglossinae (Hymenoptera, Apidae) em florestas contínuas de terra firme na Amazônia central, Brasil. Revista Brasileira de Zoologia 12: 547-556.

Pearson, D. L. \& R. L. Dressler. 1985. Two-Year Study of Male Orchid Bee (Hymenoptera: Apidae: Euglossini) Attraction to Chemical Baits in Lowland South-Eastern Peru. Journal of Tropical Ecology 1: 37-54.

Peruquetti, R. C.; L. A. O. Campos; C. D. P. Coelho; C. V. M. Abrantes \& L. C. O. Lisboa. 1999. Abelhas Euglossini (Apidae) de áreas de Mata Atlântica: abundância, riqueza e aspectos biológicos. Revista Brasileira de Zoologia 16: 101-118.

Powell, A. H. \& V. N. Powell. 1987. Population dynamics of male euglossine bees in Amazonian forest fragments. Biotropica 19: $176-179$.

Raw, A. 1989. The dispersal of euglossine bees between isolated patches of eastern Brazilian wet Forest (Hymenoptera, Apidae). Revista Brasileira de Entomologia 33: 103-107.

Rebêlo, J. M. M. \& A. J. M. Cabral. 1997. Abelhas Euglossinae de Barreirinhas, zona do litoral da Baixada Oriental Maranhense. Acta Amazonica 27: 145-152.

Rebêlo, J. M. M. \& C. A. Garófalo. 1991. Diversidade e sazonalidade de machos de Euglossini (Hymenoptera, Apidae) e preferências por iscas-odores em um fragmento de floresta no sudoeste do Brasil. Revista Brasileira de Biologia 51: 787-799.

Rebêlo, J. M. M. \& C. A. Garófalo. 1997. Comunidades de Machos de Euglossini (Hymenoptera: Apidae) em matas semidecíduas do nordeste do Estado de São Paulo. Anais da Sociedade Entomológica do Brasil 26: 243-255.

Rebêlo, J. M. M. \& F. S. Silva FS. 1999. Distribuição das abelhas Euglossini (Hymenoptera, Apidae) no Estado do Maranhão, Brasil. Anais da Sociedade Entomológica do Brasil 26: 379-391.

Ricklefs, R. E.; R. M. Adams \& R. L. Dressler. 1969. Species diversity of Euglossa in Panama. Ecology 50: 713-716.

Rocha, C. F. D.; H. G. Bergallo; M. A. S. Alves \& M. V. Sluys. 2003. A biodiversidade nos grandes remanescentes florestais do Estado do Rio de Janeiro e nas restingas da Mata Atlântica. Rima Editora, São Carlos, 163 p.

Roubik, D. W. 1989. Ecology and natural history of tropical bees. Cambridge University Press, Cambridge, 514 p.

Roubik, D. W. \& Ackerman J. D. 1987. Long-term ecology of euglossine orchid-bees (Apidae: Euglossini) in Panama. Oecologia 73: 321333.

Roubik, D. W. \& P. E. Hanson. 2004. Orchid bees of tropical America. Biology and field guide. INBIO, Santo Domingo de Heredia: Costa Rica. 352 pp.

Silva, F. S. \& J. M. M. Rebêlo. 2002. Population dynamics of Euglossinae bees (Hymenoptera, Apidae) in an early second-growth forest of Cajual island, in the State of Maranhão, Brazil. Brazilian Journal of Biology 62: 15-23.

Singer, R. B. 2003. Orchid pollination: recent developments from Brazil. Lankesteriana 7: 111-114.

Sofia, S. H. \& K. M. Suzuki. 2004. Comunidades de abelhas Euglossina (Hymenoptera: Apidae) em fragmentos florestais no sul do Brasil. Neotropical Entomology 33: 693-702.

Souza, K. P.; M. I. M. Hernández \& C. F. Martins. 2005. Riqueza, abundância e diversidade de Euglossina (Hymenoptera, Apidae) em três áreas da Reserva Biológica Guaribas, Paraíba, Brasil. Revista brasileira de Zoologia 22: 320-325.

Tonhasca, A.; J. L. Blackmer \& G. S. Albuquerque. 2002. Abundance and diversity of euglossine bees in the fragmented landscape of the brazilian Atlantic Forest. Biotropica 34: 416-422.

Tonhasca Jr.; A. G. S. Albuquerque \& J. L. Blackmer. 2003. Dispersal of euglossine bees between fragments of the brazilian Atlantic Forest. Journal of Tropical Ecology 19: 990-102.

Whittaker, R. H. 1965. Dominance and diversity in land plant communities. Science 147: 250-260

Wolda, H. 1981. Similarity indices, sample size and diversity. Oecologia 50: 296-302. 